\title{
Strength of object representation: its key role in object-based attention for determining the competition result between Gestalt and top-down objects
}

\author{
Jingjing Zhao ${ }^{1,2} \cdot$ Yonghui Wang ${ }^{1,2} \cdot$ Donglai Liu $^{3} \cdot$ Liang Zhao ${ }^{1,2} \cdot$ Peng Liu ${ }^{1,2}$
}

Published online: 4 June 2015

(C) The Psychonomic Society, Inc. 2015

\begin{abstract}
It was found in previous studies that two types of objects (rectangles formed according to the Gestalt principle and Chinese words formed in a top-down fashion) can both induce an object-based effect. The aim of the present study was to investigate how the strength of an object representation affects the result of the competition between these two types of objects based on research carried out by Liu, Wang and Zhou [(2011) Acta Psychologica, 138(3), 397-404]. In Experiment 1, the rectangles were filled with two different colors to increase the strength of Gestalt object representation, and we found that the object effect changed significantly for the different stimulus types. Experiment 2 used Chinese words with various familiarities to manipulate the strength of the topdown object representation. As a result, the object-based effect induced by rectangles was observed only when the Chinese word familiarity was low. These results suggest that the strength of object representation determines the result of competition between different types of objects.
\end{abstract}

Keywords Object-based effect - Object representation strength $\cdot$ Chinese word familiarity

Jingjing Zhao and Yonghui Wang contributed equally to this work.

Yonghui Wang

wyonghui@snnu.edu.cn

1 School of Psychology, Shaanxi Normal University, 199, South Chang'an Road, Yanta District, Xi'an 710062, China

2 Shaanxi Provincial Key Laboratory of Behavior \& Cognitive Neuroscience, Xi'an, China

3 Shaanxi Regional Electric Power Group Co., Xi'an, China

\section{Introduction}

Attention is the ability to concentrate on relevant information in an environment while ignoring other information. This ability is restricted not only by space (Posner, Snyder, \& Davidson, 1980; Hoffman \& Nelson, 1981), but also by the number of attended objects (Neisser, 1967; Kahneman \& Henik, 1981; Treisman, Kahneman, \& Burkell, 1983). Egly, Driver, and Rafal (1994) presented participants with two parallel rectangles, one end of which was brightened as a cue. The target was a solid square emerging at the cued end (valid), the other end of the cued rectangle (invalid same object), or an equidistant end of the uncued rectangle (invalid different object). The results showed that reaction times (RTs) were shortest in the valid condition. Crucially, RTs were shorter in the invalid same object condition than in the invalid different object condition, which was considered evidence of an objectbased effect. This result also indicated that there would be a time cost when switching attention between objects.

The objects formed by many other Gestalt principles can also induce object-based effects (such as that found by Egly et al. 1994; Falter, Grant, \& Davis, 2010; Festman \& Braun, 2012; Harrison \& Feldman, 2009; Kasai, 2010; Moore, Yantis, \& Vaughan, 1998; Lavie \& Driver, 1996; Behrmann, Zemel, \& Mozer, 2000). Moreover, top-down information can also affect object-based attention. In some cases, top-down information has been used to change subjects' strategy and could influence their attention deployment by overriding objects (Peterson \& Hochberg, 1983; Shomstein \& Behrmann, 2008; Ho \& Yeh, 2009). In other cases, top-down objects could be defined directly by subjects' lexical knowledge as such lexical-based objects can constrain attention deployment in the same way as perceptual-based objects (Liu, Wang, \& Zhou, 2011; Robertson \& Treisman 2006; Vecera \& Farah, 1997; Yuan \& Fu, 2014). Li and Logan (2008) used Chinese 
words to investigate the object-based effect in visual attention. They presented subjects with two pairs of Chinese words, each of which contained two Chinese characters. The two words were parallel horizontally or vertically and they never made sense when combined together. The cue was one character and the target was either the cued character (valid), the other character in the cued word (invalid same word), or another character adjacent to the cued character in the uncued word (invalid different word). The results showed that RTs were shorter when the cued and target characters were in the same word than when they were in different words. This result suggested that the objects formed in a top-down fashion could also constrain attention deployment. The paradigm used in this research is similar to the one used by Egly et al. (1994). The difference is that, in the study of Li and Logan (2008), the objects were Chinese words formed by top-down lexical information instead of rectangles formed by a bottom-up Gestalt principle.

Given that objects can be formed either by the Gestalt principle or in a top-down fashion, and that we live in an environment filled with both top-down and bottom-up objects, an interesting question is how the attentional system makes sense of the competing effects of these two types of objects. This question is important because the pattern of effects allows us to infer how the cognitive processes underlying the two types of object effects work together in constraining attentional shift and the extent to which the functioning of the cognitive processes is affected by task demands or contextual factors. To answer this question, two mechanisms have been proposed (Liu et al., 2011; Shomstein \& Behrmann, 2008; Shomstein \& Yantis, 2004; Yeari \& Goldsmith, 2010). On the one hand, it is possible that the attentional system selects one type of object while attenuating the other. This would result in a pattern of data that would reflect only one type of object-based effect such that an improvement in RT would occur only when the target appears within the dominant object. In an adapted double-rectangle paradigm (Egly et al., 1994), Shomstein and Yantis (2004) and Shomstein and Behrmann (2008) manipulated top-down fashion by varying the probability that a target would appear in each of two uncued locations. On the invalidly cued trials, the target appeared in the highprobability location (defined by an absolute spatial location, e.g., upper right) $83 \%$ of the time and in the low-probability location (e.g., lower left) $17 \%$ of the time. In half of the invalidly cued trials, the high-probability location was the invalid-same object location, and the low-probability location was the invalid-different object location (congruent). In another half of the invalidly cued trials, the high-probability location was the invalid-different object location, and the lowprobability location was the invalid-same object location (conflict). The results showed that, in the conflict condition, the bottom-up object-based effect disappeared and the RTs depended exclusively on the top-down effect. Yeari and
Goldsmith (2010) found similar results using an adapted spatial cuing paradigm.

On the other hand, the processes subserved by the two types of object structures might put the attentional shift in limbo and thereby eliminate the overall object effect. In this case, the attentional system may not be able to use either the top-down or bottom-up fashion, resulting in no difference in RT across the two invalid locations. Recently, Liu et al. (2011) presented subjects with two parallel Chinese two-character words and two rectangles simultaneously. In some trials of their experiment, the orientation of the words was orthogonal to that of the rectangles. That is, when the cue and target were in the same words, they were in different rectangles and vice versa. In their research, if one type of object could induce an object-based effect by overriding the other, the RTs would be shorter when the cue and target were in the same word (Chinese words override rectangles) or in the same rectangle (rectangles override Chinese words). However, the results showed that the object-based effect was not observed when these two types of objects were competitive.

However, there are three possible shortcomings of the existing studies (Liu et al. 2011; Shomstein \& Behrmann, 2008; Shomstein \& Yantis, 2004; Yeari \& Goldsmith, 2010). First, the top-down mode used in the previous studies, such as experimental instructions, short-term experience or strategies like target-location probability, was used to facilitate target search rather than to form objects (Chen, 1998; Shomstein \& Behrmann, 2008; Shomstein \& Yantis, 2004; Yeari \& Goldsmith, 2010; Zemel, Behrmann, Mozer, \& Bavelier, 2002). In this condition, the competing processing between top-down and bottom-up objects could not be observed directly. Second, most studies found that top-down objects could always override Gestalt principle objects to dominate attention deployment. However, we assumed that this result might be due to the fact that the strength of the top-down object representations used in those studies was higher than that of the Gestalt principle object. Third, in the previous two mechanisms, the attentional system may not be able to actively generate accurate anticipations. Specifically, the attentional system may not be able to automatically ignore dominant objects or use either object alternately. In our opinion, both top-down and bottom-up information can be used to impact visual perception such that either a top-down or bottom-up object-based effect can be found based on the strength of object representation. That is, given the apparent flexibility of the attentional system, which mode could dominate attention deployment when the two effects existed simultaneously was determined by competition between the strength of their representations; the stronger could override the weaker and thus play a leading role in attention deployment. Essentially, we argue that our attentional system is able to select object dominance based on the strength of object representations and can alternate 
flexibly between entraining the top-down or bottom-up object-based effect.

Therefore, the present study used objects that were formed directly by top-down lexical information, and manipulated the strength of the Gestalt and top-down object representations. We thus investigated whether the strength of an object representation was the key factor influencing the competition result when the two types of objects were competitive. To answer this question, we conducted two experiments. In Experiment 1 , we filled the rectangles with red and blue to strengthen the Gestalt object representation, but this manipulation could also be considered as adding an object formed by color to the rectangles. To resolve that, in Experiment 2, we classified Chinese words into those with high word familiarity and those with low word familiarity. In this way, we could compare the strength of representations of these two types of objects without the additive influence of the problem that possibly existed in Experiment 1.

\section{Experiment 1}

Liu et al. (2011) found no interaction between stimulus type and cue validity when Gestalt and top-down objects were competitive. However, the RTs were somewhat shorter when the target and cue compounded a word than a non-word, although the difference was not statistically significant. This result might be caused by the similarity in strength between the representations of the two types of objects used in their research such that neither could individually dominate attention deployment. Therefore, we planned to increase the strength of representation of one object to make it more advantageous for competition with the other. Shomstein \& Behrmann (2008) strengthened the rectangles' representations by filling them with red and blue. They found that when the target appeared in a location with high probability, RTs were always shorter, regardless of whether the target and cue were in the same object. However, when they filled the rectangles with two different colors, the object-based effect was regained under the same target appearing probability. Experiment 1 used the same method to enhance the strength of rectangles' representations.

\section{Method \\ Participants}

Twenty-one native speakers of Chinese from Shannxi Normal University were paid to participate in Experiment 1. All of them were right handed and had normal or corrected-tonormal vision.

\section{Design and materials}

A 3 (stimulus type: compound only, incongruent, rectangle with non-words) $\times 3$ (cue validity: valid, invalid same object, invalid different objects) within-subjects factorial design was used. Stimulus type was defined by the relationship between the rectangles and words presented, as shown in Fig. 1. The two Chinese words were either presented alone in the "compound only" condition or between rectangles in the "incongruent" condition. Finally, the four characters forming non-words were presented in rectangles in the "rectangle with non-words" condition. Cue validity was defined by the relative location between the cue and target. In the valid condition, the cue and target were the same character. The invalid same object condition contained two situations. One situation was that the target was another character in the cued word when there were words presented. The other situation was that the

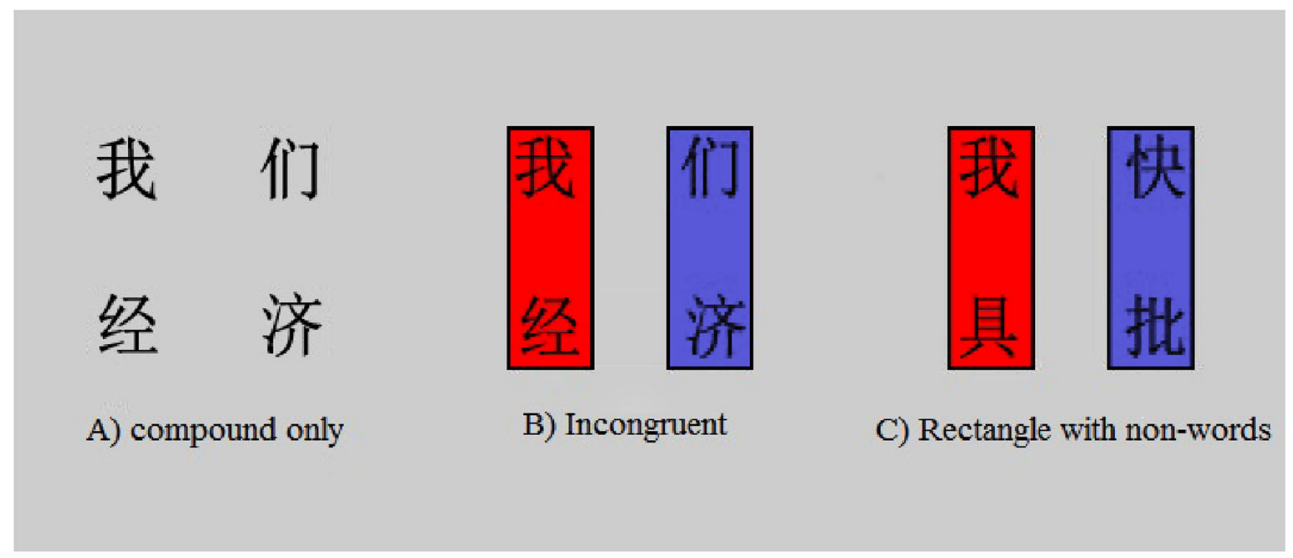

Fig. 1 a-c The three types of stimuli used in Experiment 1. a Compound only: only compound words were presented. b Incongruent: the two characters of a compound were presented separately in the two rectangles; the orientation of the words (horizontal in the example) was orthogonal to that of the rectangles (vertical in the example). $\mathbf{c}$ Rectangle with non-words: the four characters could not form any compound word in any direction 
target was another character in the cued rectangle when there were no words presented (i.e., in the "non-word" condition). The invalid different object condition included the similar two situations as did the invalid same object condition, except the target and cue were adjacent in different words (or rectangles). Combinations of stimulus type and cue validity were counterbalanced across orientation (horizontal and vertical) and cue location (upper left, upper right, lower left, lower right).

There were 888 trials in total, which took about $1.5 \mathrm{~h}$ for the participants to complete, with 768 critical trials having a target and 120 catch trials without the target. For the critical trials, there were 384 (50\%) trials for valid cueing and 192 (25\%) trials for each of the two invalid conditions. Each stimulus type has 296 trials, which were mixed randomly and divided into four blocks equally.

In total, 888 different pairs of Chinese two-character words were chosen in Experiment 1, and these were divided into three sets, one set for each stimulus type. The sets were counter-balanced across the participants. Especially, for the "rectangle with non-words" condition, the 1184 characters forming 592 different Chinese words were randomized to ensure that the four characters used in each trial never composed any word.

The frequencies of the 1776 words were from 16 to 860 per million words, with an average frequency of 77 . The number of character strokes was between 3 and 12 for low visual complexity. In each trial, the four characters were different.

\section{Procedures}

Stimuli were presented on a 21 -in CRT monitor $(1024 \times 768$ resolution; frame rate $100 \mathrm{~Hz}$ ) controlled by a Lenovo PC. A chinrest located $57 \mathrm{~cm}$ from the monitor was used to minimize head movements. Each character subtended $0.8 \times 0.8^{\circ}$ of visual angle (mean luminance $=24.30 \mathrm{~cd} \mathrm{~m}^{-2}$ ) and the distance between the centers of two adjacent characters was $1.6^{\circ}$ of visual angle. Each rectangle subtended $0.9^{\circ}$ in width and $2.5^{\circ}$ in length and its outline width was approximately $0.05^{\circ}$ of visual angle. The two rectangles were filled with red (RGB: $255,0,0)$ (luminance $=27.79 \mathrm{~cd} \mathrm{~m}^{-2}$ ) and blue (RGB: 0, 0, 255) (luminance $=23.18 \mathrm{~cd} \mathrm{~m}^{-2}$ ), respectively. Eye movements were recorded with an Eye-Link 2 tracker (with a sampling rate of $250 \mathrm{~Hz}$ ). All the characters were shown in black against a gray background (luminance $=31.86 \mathrm{~cd} \mathrm{~m}^{-2}$ ). The fixation was a plus sign $(+)$, subtended $0.1^{\circ} \times 0.1^{\circ}$ of visual angle.

Each trial began with a display of a stimulus set consisting of four Chinese characters for $1500 \mathrm{~ms}$ (Fig. 2), followed by a plus sign $(+)$ in addition to the characters at the center of the display for $300 \mathrm{~ms}$. Participants were asked to maintain fixation on the plus sign from then on. One character turned green as a cue for $100 \mathrm{~ms}$ and turned black after the cue for another $100 \mathrm{~ms}$. After this stimulus onset asynchrony (SOA) of $200 \mathrm{~ms}$, a character was colored in white and participants were asked to press a button on a joystick as soon

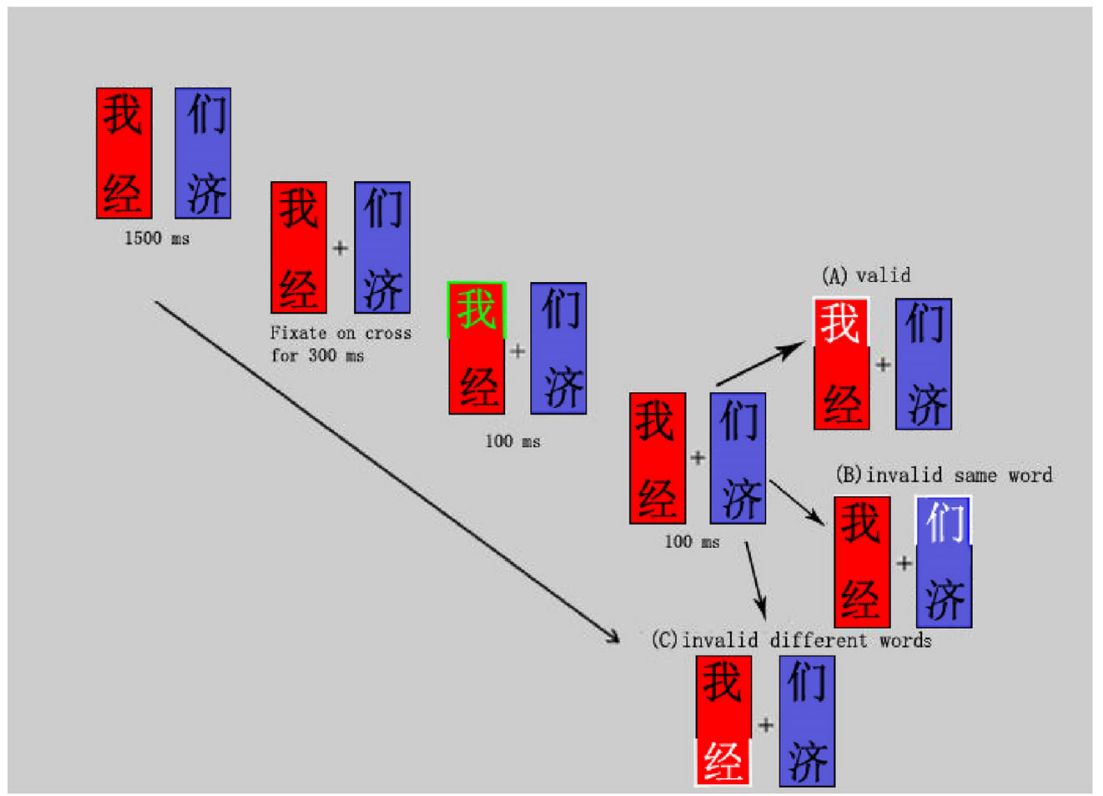

Fig. 2 a-c Sequence of events in a single trial in Experiment 1. Four Chinese characters forming two compound words ("we" and "economic" in the example) were presented, and the participants were asked to press a response key when they detected the presence of a white color target after having seen a green color cue. a A valid trial: the color cue and the color target were on the same character. b An "invalid same word" trial: the cue character and the target character formed a compound word ("we"). c An "invalid different words" trial: the target was another character adjacent to the cue character, but these two characters formed a non-word 
as they detected the presence of the white color or to do nothing if no character turned white. After the button press (or after $1500 \mathrm{~ms}$ if there was no response), the eye tracking system performed drift correction, and the experimenter pressed the space bar connected with the control computer to start the next trial. A trial was aborted and a text line ("you moved your eyes") was shown on the screen for $1000 \mathrm{~ms}$ if participants did not maintain fixation on the plus sign. A text line reading "incorrect response" was shown on the screen for $1000 \mathrm{~ms}$ when participants made a false response to the catch trial or did not respond to the target within 1500 ms. Participants underwent a training block of 48 trials before being formally tested.

\section{Results}

\section{Eye movements}

Participants made eye movements (leaving the fixation area, which was $0.8 \times 0.8^{\circ}$ in visual angle) in $4.4 \%$ (ranging between $0.6 \%$ and $11.5 \%$ ) of all trails. These trials were excluded from further analysis. The percentage of excluded trials did not vary between conditions.

\section{Manual responses}

The hit rate (for critical trials) and false alarm rate (for catch trials) were $99.9 \%$ and $4.3 \%$, respectively. Reaction times (RTs) shorter than $150 \mathrm{~ms}$ were removed, removing $0.1 \%$ of the total critical trials. RTs that were more than three standard deviations away from the overall mean across conditions for that participant were also discarded, removing another $1.1 \%$ of the critical trials.

A two-way repeated-measures analysis of variance (ANOVA) was conducted, with stimulus type and cue validity as two within-subjects factors. The main effect of stimulus type was not significant $\left[\mathrm{F}(2,40)=.45, P=.64, \eta_{\mathrm{p}}{ }^{2}=.02\right]$. The main effect of cue validity was significant $[\mathrm{F}(2,40)=$ $\left.28.82, P<.01, \eta_{\mathrm{p}}{ }^{2}=.59\right]$ ]. Importantly, the interaction between stimulus type and cue validity was significant $[\mathrm{F}(4,80)=$ $\left.18.37, P<.01, \eta_{\mathrm{p}}{ }^{2}=.48\right]$. More specifically, the RTs were shortest when the target was presented on the cued location in each stimulus type (all $P$ values $<.05$ ), indicating a space-based effect. However, a planned test showed that the RT difference between "invalid different objects" and "invalid same objects" was significant in the "compound only" condition $(\mathrm{M}=4 \mathrm{~ms} ; \mathrm{SE}=1.63)[\mathrm{F}(1,20)=5.94$, $\left.P=.02, \eta_{\mathrm{p}}{ }^{2}=.23\right]$ and in the "rectangle with non-words" condition $(\mathrm{M}=10 \mathrm{~ms} ; \mathrm{SE}=3.15)[\mathrm{F}(1,20)=9.59, P<$ $\left..01, \eta_{\mathrm{p}}{ }^{2}=.32\right]$, but not in the "incongruent" condition (M
$=-2 \mathrm{~ms} ; \mathrm{SE}=3.94)\left[\mathrm{F}(1,20)=0.36, P=.56, \eta_{\mathrm{p}}{ }^{2}=.02\right]$ (see Fig. 3).

\section{Discussion}

In Experiment 1, we filled the rectangles with two different colors to make their representations more prominent. In this way, we tried to enhance the representations of the rectangles and give them an advantage in the competition with Chinese words. However, attention deployment was still not dominated by the rectangles when the two types of objects were arranged competitively because neither a top-down nor a bottom-up object-based effect was observed in the "incongruent" condition. This result was similar to that obtained by Liu et al. (2011). However, it seems likely that in our Experiment 1, when the rectangles' representations were strengthened by filling them with different colors, the RTs were shorter when the cue and the target were in the same rectangle than when they were in different rectangles, despite Chinese words. To confirm this hypothesis, we asked an additional 13 participants to perform a task similar to that in Experiment 1, except that there was only an "incongruent" condition and the rectangles could be filled (in our Experiment 1) or unfilled (Liu et al. 2011) with colors. A two-way repeated-measures ANOVA was conducted, with rectangle type (filled with colors vs unfilled) and cue validity as two within-subjects factors, RT as the dependent measure. The main effect of rectangle type was not significant $[\mathrm{F}$ $\left.(1,12)=.22, P=.65, \eta_{\mathrm{p}}^{2}=.02\right]$. The main effect of cue validity was marginally significant $[\mathrm{F}(2,24)=3.05, P=$ $\left..06, \eta_{\mathrm{p}}{ }^{2}=.20\right]$. Importantly, the interaction between rectangle type and cue validity was significant $[\mathrm{F}(2,24)=$

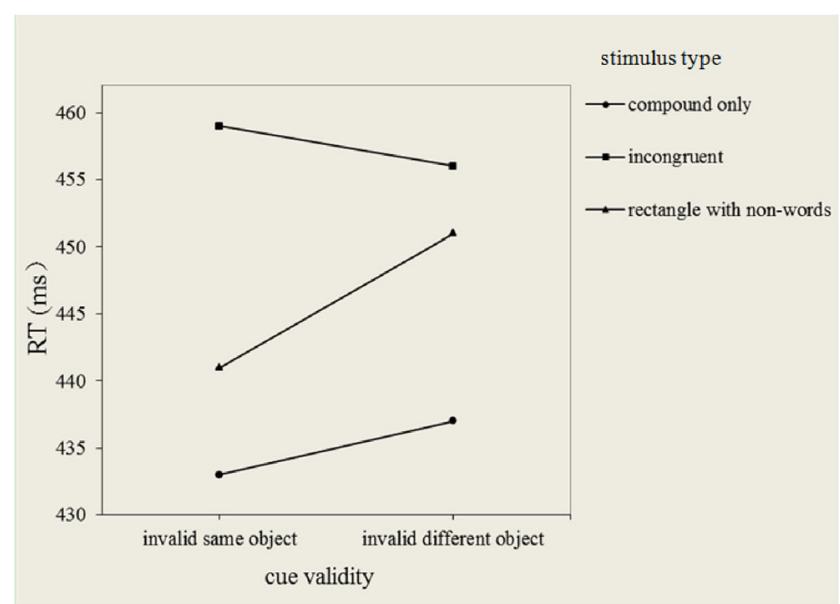

Fig. 3 Mean reaction times (RTs) for the invalidly cued trials from Experiment 1 for the compound only, incongruent and rectangle with nonwords conditions 
7.00, $\left.P<.01, \eta_{\mathrm{p}}{ }^{2}=.37\right]$. Moreover, in order to explore whether the object-based result observed in the "rectangles filled with colors" condition was different from that observed in the "rectangles unfilled with colors" condition, we performed a 2 (rectangle type: filled with colors vs unfilled) $\times 2$ (cue validity: invalid same object vs invalid different objects) within-subjects factorial design. The results showed a significant interaction between rectangle type and cue validity $[\mathrm{F}(1,12)=9.63, P=.01$, $\left.\eta_{\mathrm{p}}{ }^{2}=.45\right]$. A planned test showed that the RT difference between the "invalid same object" and "invalid different objects" was negative in the "rectangles filled with colors" condition $(\mathrm{M}=-13 \mathrm{~ms}, \mathrm{SE}=4.45)[\mathrm{F}(1,12)=$ 9.13, $\left.P=.01, \eta_{\mathrm{p}}{ }^{2}=.28\right]$, but was not significant in the "rectangles unfilled with colors" condition $(\mathrm{M}=3 \mathrm{~ms}$, SE $=3.88)\left[\mathrm{F}(1,12)=.66, P=.43, \eta_{\mathrm{p}}{ }^{2}=.05\right]$.

In summary, when we filled the rectangles with different colors to strengthen their representations, they had an advantage in the competition with Chinese words. However, this manipulation could also be regarded as adding a colored object on top of another object that is made of a geometric shape. According to this possibility, our results obtained in Experiment 1 might be due to the two types of Gestalt principle objects congruently competing with the Chinese words. Therefore, in the subsequent experiment, we varied the strength of the Chinese word representations without changing their physical attribution to see whether a similar result would be obtained.

\section{Experiment 2}

Vecera and Farah (1997) presented two overlapping transparent shapes in the center of the screen, the shapes might be upright letters, letters that were rotated by $180^{\circ}$, or non-letter shapes. Participants were asked to determine whether the two Xs were on the same shape or on different shapes. The results showed that RTs were shorter when the targets appeared in a familiar upright letter than when they appeared in a less-familiar, rotated letter. Additionally, Robertson and Treisman (2006) presented "NO" and "ON" or "ZO" and "OZ" to the participants, and asked them to report whether the "N" or "Z" was to the right or to the left of the "O". They found that the familiar words (NO or ON) allowed participants to form objects more than unfamiliar words ( $\mathrm{ZO}$ or $\mathrm{OZ}$ ). These results suggested that letter familiarity influences object-based attention. According to these findings, we changed the strength of Chinese word representations by varying their lexical familiarity to determine whether the attention deployment would be influenced by the strength of the contrast of Gestalt and top- down objects. Moreover, the lexical familiarity manipulation avoided the problems of the objects overlapping that occurred in Experiment 1.

\section{Method}

\section{Participants}

Twenty native speakers of Chinese from Shannxi Normal University were paid to participate in Experiment 2. All were right handed and had normal or corrected-to-normal vision; none of them had participated in Experiment 1.

\section{Design and materials}

A 2 (word familiarity: familiar, unfamiliar) $\times 3$ (cue validity: valid, invalid same object, invalid different objects) withinsubjects factorial design was used. Each Chinese word familiarity was evaluated by the subjects via a seven-point Likert measurement. Based on this evaluation, word familiarity was defined by the average Likert value of the two Chinese words shown in each trial. In the "familiar" condition, the two Chinese words had high Likert values, whereas those with low Likert values were assigned to the "unfamiliar" condition. Cue validity had the same definition as it did in Experiment 1 . The two Chinese words were between rectangles, which was the equivalent of the "incongruent" condition in Experiment 1. Combinations of word familiarity and cue validity were counterbalanced across orientations and cue locations.

There were 592 trials in total, which took about $1 \mathrm{~h}$ for the participants to complete, with 512 critical trials having a target and 80 catch trials without the target. For the critical trials, there were $256(50 \%)$ valid trials and 128 trials $(25 \%)$ for each of the two invalid conditions. All the trials were randomly mixed.

We selected the Chinese words as follows. First, we selected 768 high frequency Chinese words with a frequency ranging from 63 to 995 per million words (the average frequency was 225 per million words). Then, another 768 low-frequency Chinese words were selected with frequencies from 0.8 to 8 per million words. Chinese words with a frequency of 0.8 comprised $95 \%$ of all of the low-frequency words. Second, we asked subjects who did not participate in the formal experiment to evaluate the familiarity of all the 1536 Chinese words. Because of the large number of Chinese words needed to be evaluated by a subject at one time, we divided the 768 high-frequency and 768 low-frequency Chinese words into two equal parts, which were called parts $\mathrm{A}$ and $\mathrm{B}$ and parts $\mathrm{C}$ and $\mathrm{D}$, respectively. On that basis, we compounded parts $\mathrm{A}$ and $\mathrm{C}, \mathrm{B}$ and $\mathrm{C}, \mathrm{A}$ and $\mathrm{D}$, and $\mathrm{B}$ and $\mathrm{D}$ into four sets, and the words were mixed randomly in each set. Then, each subject was asked to evaluate the familiarity of all Chinese words in 
one set on a 7-point Likert scale from " 1 " = very unfamiliar to " $7 "=$ very familiar. Every set was evaluated by 15 subjects, in other words, each word was evaluated by 30 subjects in total. Third, we ranked the 768 high-frequency Chinese words in descending order based on familiarity, and the top 592 words were selected. Similarly, we ranked the 768 low-frequency Chinese words in ascending order based on familiarity, and the top 592 words were selected. Their familiarities were from 4.7 to 6.9 and 1.6 to 4.8 , respectively. The Chinese words with familiarities above 6 accounted for $96 \%$ of the 592 familiar words, and words with familiarities below 4.5 for $94 \%$ of the 592 unfamiliar words. An independent $t$ test showed a significant difference in familiarity between the familiar and unfamiliar words $[t(1182)=-98.09, P<.001]$. Finally, pairs of words with similar high or low familiarities were used in Experiment 2.

\section{Procedures}

The procedure was the same as in Experiment 1, except the two rectangles in each trial were no longer filled with colors and the target was shown in red.

\section{Results}

\section{Eye movements}

Participants made eye movements (leaving the fixation area, which was $0.8 \times 0.8^{\circ}$ of visual angle) in $3.2 \%$ (ranging between $0.5 \%$ and $11.3 \%$ ) of all trials. These trials were excluded from further analysis. The percentage of excluded trials did not vary between conditions.

\section{Manual responses}

The hit rate (for critical trials) and false alarm rate (for catch trials) were $99.9 \%$ and $3.4 \%$, respectively. RTs shorter than $150 \mathrm{~ms}$ were removed, accounting for $0.2 \%$ of all critical trials. RTs that were more than three standard deviations away from the overall mean across conditions for that participant were also discarded, accounting for another $1.0 \%$ of the critical trials.

A two-way repeated-measures ANOVA was conducted, with word familiarity and cue validity as two within-subjects factors. The main effect of word familiarity was significant $[\mathrm{F}$ $\left.(1,19)=4.88, P=.04, \eta_{\mathrm{p}}{ }^{2}=.21\right]$, indicating that the familiar words $(\mathrm{M}=373 \mathrm{~ms} ; \mathrm{SE}=11.02)$ were of more benefit for participants in their response than unfamiliar words $(\mathrm{M}=$ $379 \mathrm{~ms}$; $\mathrm{SE}=11.99)$. The main effect of cue validity was also significant $\left[\mathrm{F}(2,38)=9.42, P<.01, \eta_{\mathrm{p}}{ }^{2}=.33\right]$. More specifically, the RTs were shortest when the target was presented on the cued location in each word familiarity condition (both $P$ values $<.05$ ), indicating a space-based effect. The interaction between word familiarity and cue validity was not significant $\left[\mathrm{F}(2,38)=2.66, P=.08, \eta_{\mathrm{p}}{ }^{2}=.12\right]$. However, we further explored whether the object-based result was significantly different between "familiarity" and "unfamiliarity" conditions. Thus, we performed another 2 (word familiarity: familiar, unfamiliar) $\times 2$ (cue validity: invalid same object, invalid different objects) within-subjects factorial design. The ANOVA revealed a significant interaction $\left[\mathrm{F}(1,19)=5.53, P=.03, \eta_{\mathrm{p}}{ }^{2}=\right.$ .23]. A planned test showed that the RT difference between the "invalid different objects" and the "invalid same objects" was not significant in the "familiar" condition $(\mathrm{M}=3 \mathrm{~ms}$; $\mathrm{SE}$ $=2.53)\left[\mathrm{F}(1,19)=1.53, P=.23, \eta_{\mathrm{p}}{ }^{2}=.08\right]$. But interestingly, RTs to the "invalid different objects" trials were even shorter than those to the "invalid same objects" trials in the "unfamiliar" condition $(\mathrm{M}=-4 \mathrm{~ms}$; $\mathrm{SE}=1.71)[\mathrm{F}(1,19)=$ $\left.4.87, P=.04, \eta_{\mathrm{p}}{ }^{2}=.20\right]$, indicating that the bottom-up objectbased effect was induced by the rectangles in the "unfamiliar" condition (see Fig. 4).

To reveal how the words' familiarity influenced the trend in the object-based effect, we conducted two correlation analyses in which one factor was the trial RT, an average RT for each trial across the 20 subjects, and the other factor was the trial familiarity, an average of the two words' familiarities evaluated by the 20 subjects participating in the formal experiment.

In the "invalid same object" condition, the normal distribution test for the two factors showed that the "trial RT" fit a normal distribution ( statistic $=.06, \mathrm{df}=128, P=.20$ ) but that "trial familiarity" did not (statistic $=.31, \mathrm{df}=128, P<.01)$. As a result, we conducted a Spearman correlation analysis, which showed that there was a marginally significant negative correlation between "trial RT" and "trial familiarity" (Spearman correlation $=-.15, P=0.09)$, indicating that the subjects could group the cue and target into one Chinese word more easily when the word was familiar, thus leading to a faster response to the target.

In the "invalid different object" condition, the Spearman correlation analysis for "trial RT" and "trial familiarity" showed that there was a marginally significant positive correlation (Spearman correlation $=.16, P=0.07$ ), indicating that the subjects could distinguish that the cue and target were not in a Chinese word more easily when the word was familiar, which led to a slower response to the target.

\section{Discussion}

In Experiment 2, when the Chinese word was unfamiliar, a bottom-up object-based effect induced by the rectangles was found due to the weak strength of the top-down object representations. This result suggested that the Gestalt rectangles could dominate attention deployment if the strength of the top-down object representations were sufficiently weak. 


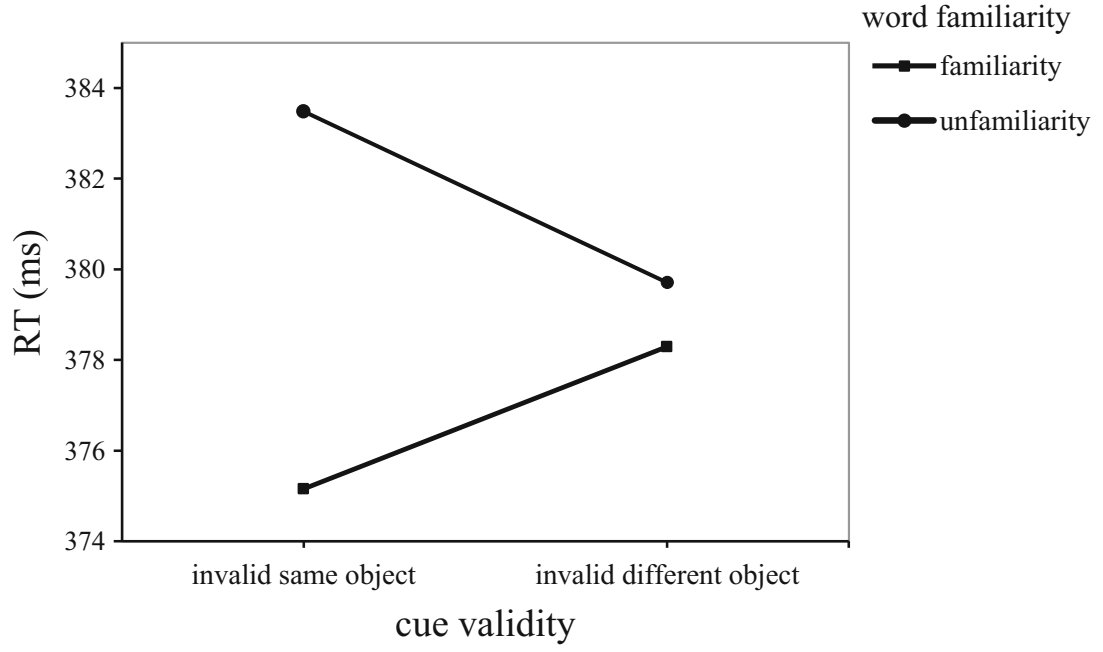

Fig. 4 Mean RTs for the invalidly cued trials from Experiment 2 for the familiarity and unfamiliarity conditions

Furthermore, the correlation analysis results showed that when word familiarity increased, RT was shorter when the cue and target compounded to form a Chinese word and longer when the cue and target compounded to form a non-word. Both results suggest that the top-down or bottom-up same object advantage would be more prominent if the object representation of the appropriate category had an advantage in the competition with the other.

\section{General discussion}

In the present study, we investigated the key factor for determining the result of competition between top-down (Chinese words) and Gestalt principle (rectangles) objects. In Experiment 1, we strengthened the rectangles' representations by filling the shapes with different colors, showing that, relative to Chinese words, attention deployment tended to be more affected by the rectangles. Based on this finding, in Experiment 2, we changed the strength of Chinese word representations by selecting words with various familiarities. In this way, the possible overlapping problem existing in Experiment 1 could be avoided. We found that the rectangles could induce a bottom-up object-based effect when the competitive Chinese words were unfamiliar. Furthermore, the RTs to the target in those invalid conditions changed linearly as the word familiarity changed. These findings suggest that the strength of the contrast between the representations of these two types of objects determined the result of their competition, and attention deployment tended to be more affected by the type of object that had greater strength.

Some previous studies have focused on elucidating the mechanisms that give rise to an object-based effect when top-down and bottom-up objects exist simultaneously (Ho,
2011; Shomstein \& Behrmann, 2008; Shomstein \& Yantis, 2004; Yeari \& Goldsmith, 2010; Vecera, 2000). However, there are still three issues that need to be considered. First, in these studies, the results that no bottom-up object-based effects were found and that the experiment task was finished more efficiently when the subjects used a top-down strategy in the high target-location probability condition might not be because Gestalt principle objects could always be overwritten by top-down strategy. The results might be due to the fact that the strength of top-down object representations used in those studies was higher than that of Gestalt principle objects. Second, the top-down mode such as experiment instructions, short-term experience and target-location probability may have either changed the subjects' perception-set or driven them to finish the experimental task in a certain manner such that they could not form objects directly. Third, the mechanisms proposed in the previous studies ignore the initiative of the attentional system. Specifically, the attentional system could not automatically ignore dominant objects or alternately use either object. Therefore, in our study, we used Chinese words that could form top-down objects directly. Accordingly, the extent to which a top-down mode influenced attention deployment could be answered. The results showed that when Chinese words were presented alone, an objectbased effect was found. However, when the words competed with the color-filled rectangles, this effect disappeared. This might be caused by the similar strengths of the representations of these two types of objects such that neither could individually dominate attention deployment. In our opinion, both topdown and bottom-up information can be used to impact visual perception, and the mode that dominates attention deployment when they exist simultaneously is determined by the competition between the strengths of their representations. The strong one can override the weak one and thus play a leading 
role in attention deployment. Essentially, we argue that our attentional system is able to select object dominance based on strength of an object representation and can alternate flexibly between entraining to top-down or bottom-up objectbased effects.

In Ariga, Yokosawa, and Ogawa (2007), no object-based effect was found in the condition in which participants were unaware of the objects (inattentional blindness), and they attributed this result to the poor object representations. According to Ariga et al. (2007), the null results of an object-based effect in the familiar condition in our Experiment 2 might be due to the poor representation of Chinese words compared with rectangles. In summary, by filling the rectangles with different colors and by using Chinese words with various familiarities, the present study manipulated the strength of the representations of these two types of objects and found that when two types of objects were competitive with each other, attention deployment will tend to be influenced more by the object representation that has a higher strength.

Acknowledgments This study was supported by a grant from Natural Science Foundation of China (31371026) to Yonghui Wang. We thank Xingshan Li and the anonymous reviewers for helpful comments on earlier versions of this work.

\section{References}

Ariga, A., Yokosawa, K., \& Ogawa, H. (2007). Object-based attentional selection and awareness of objects. Visual Cognition, 15, 685-709.

Behrmann, M., Zemel, R. S., \& Mozer, M. C. (2000). Occlusion, symmetry, and object-based attention: Reply to Saiki (2000). Journal of Experimental Psychology: Human Perception and Performance, 26, 1497-1505.

Chen, Z. (1998). Switching attention within and between objects: The role of subjective organization. Canadian Journal of Experimental Psychology, 52, 7-17.

Egly, R., Driver, J., \& Rafal, R. D. (1994). Shifting visual attention between objects and locations: Evidence from normal and parietal lesion subjects. Journal of Experimental Psychology: General, 123, 161-177.

Falter, C. M., Grant, K. C. P., \& Davis, G. (2010). Object-based attention benefits reveal selective abnormalities of visual integration in autism. Autism Research, 3(3), 128-136.

Festman, Y., \& Braun, J. (2012). Feature-based attention spreads preferentially in an object-specific manner. Vision Research, 54, 31-38.

Harrison, S. J., \& Feldman, J. (2009). Perceptual comparison of features within and between objects: A new look. Vision Research, 49(23), 2790-2799.

Ho, M. C. (2011). Object-based attention: Sensory enhancement or scanning prioritization. Acta Psychologica, 138(1), 45-51.
Ho, M. C., \& Yeh, S. L. (2009). Effects of instantaneous object input and past experience on object-based attention. Acta Psychologica, 132, 31-39.

Hoffman, J. E., \& Nelson, B. (1981). Spatial selectivity in visual search. Perception \& Psychophysics, 30, 283-290.

Kahneman, D., \& Henik, A. (1981). Perceptual organization and attention. In M. Kubovy \& J. R. Pomerantz (Eds.), Perceptual organization (pp. 181-211). Hillsdale, NJ: Erlbaum.

Kasai, T. (2010). Attention-spreading based on hierarchical spatial representations for connected objects. Journal of Cognitive Neuroscience, 22(1), 12-22.

Lavie, N., \& Driver, J. (1996). On the spatial extent of attention in objectbased visual selection. Perception \& Psychophysics, 58, 12381251.

Li, X., \& Logan, G. D. (2008). Object-based attention in Chinese readers of Chinese words: Beyond Gestalt principles. Psychonomic Bulletin \& Review, 15, 945-949.

Liu, D., Wang, Y., \& Zhou, X. (2011). Lexical-and perceptual-based object effects in the two-rectangle cueing paradigm. Acta Psychologica, 138(3), 397-404.

Moore, C. M., Yantis, S., \& Vaughan, B. (1998). Object-based visual selection: Evidence from perceptual completion. Psychological Science, 9, 104-110.

Neisser, L. (1967). Cognitive psychology. New York: Appleton-CenturyCrofts.

Peterson, M. A., \& Hochberg, J. (1983). Opposed-set measurement procedure: A quantitative analysis of the role of local cues and intention in form perception. Journal of Experimental Psychology: Human Perception and Performance, 9, 183-193.

Posner, M. I., Snyder, C. R. R., \& Davidson, B. J. (1980). Attention and the detection of signals. Journal of Experimental Psychology: General, 109, 160-174.

Robertson, L. C., \& Treisman, A. (2006). Attending to space within and between objects: Implications from a patient with Balint's syndrome. Cognitive Neuropsychology, 23, 448-462.

Shomstein, S., \& Behrmann, M. (2008). Object-based attention: Strength of object representation and attentional guidance. Perception \& Psychophysics, 70, 132-144.

Shomstein, S., \& Yantis, S. (2004). Configural and contextual prioritization in object-based attention. Psychonomic Bulletin \& Review, 11, 247-253.

Treisman, A., Kahneman, D., \& Burkell, J. (1983). Perceptual objects and the cost of filtering. Perception \& Psychophysics, 33, 527-532.

Vecera, S. P. (2000). Toward a biased competition account of objectbased segregation and attention. Brain and Mind, 1, 353-384.

Vecera, S. P., \& Farah, M. J. (1997). Is visual image segmentation a bottom-up or an interactive process? Perception \& Psychophysics, 59, 1280-1296.

Yeari, M., \& Goldsmith, M. (2010). Is object-based attention mandatory? Strategic control over mode of attention. Journal of Experimental Psychology: Human Perception and Performance, 36, 565-579.

Yuan, J., \& Fu, S. (2014). Attention can operate on semantic objects defined by individual Chinese characters. Visual Cognition, 22, 770-788. doi:10.1080/13506285.2014.916772

Zemel, R. S., Behrmann, M., Mozer, M. C., \& Bavelier, D. (2002). Experience-dependent perceptual grouping and object-based attention. Journal of Experimental Psychology: Human Perception and Performance, 28, 202-217. 